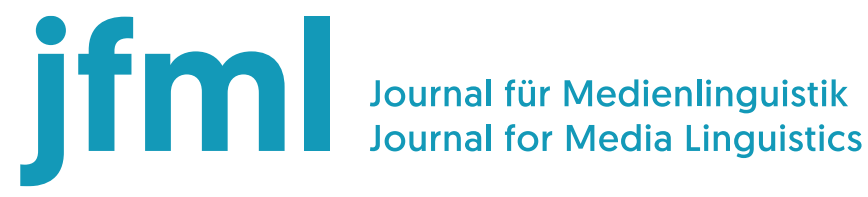

Vol 3 (2020), No 1: 14-45

DOI: $10.21248 / \mathrm{jfml} .2020 .11 .2$

Gutachten und Kommentare unter: http://dp.jfml.org/2019/opr-czulo-

nyhuis-weyell-der-einfluss-extremistischer-gewaltereignisse-auf-dasframing-von-extremismus-in-online-medien/

\section{Der Einfluss extremistischer Gewaltereignisse auf das Framing von Extremismen auf SPIEGEL Online}

Oliver Czulo \& Dominic Nyhuis \& Adam Weyell

\begin{abstract}
In diesem Beitrag untersuchen wir die Darstellung von Rechtsextremismus, Linksextremismus und Islamismus im medialen Diskurs am Beispiel von SPIEGEL Online, einem der deutschen Leitmedien. Wir leiten vier zentrale Dimensionen für die Konzeptualisierung von Extremismen ab: Ideologie und Organisation, Herkunft der Akteure, Stellung zur Gesellschaft und Typische Handlungen. Wir beobachten die Entwicklung der Darstellung der drei Extremismen an möglichen Bruchpunkten: Wir untersuchen das assoziative Framing der drei Extremismen vor und nach prominenten extremismusbezogenen Gewaltereignissen, namentlich die Anschläge des 11. September, die Veröffentlichung des NSU-Skandals und linksextremistische Aktivitäten während des G20-Gipfels in Hamburg. Mittels einer Kollokationsanalyse identifizieren wir mit den Extremismen assoziierte Aspekte und ordnen diese den Konzeptualisierungsdimensionen zu. Wir beobachten Veränderungen im Framing, die durch die ausgewählten Ereignisse bedingt sind, und vergleichen das resultierende Framing mit den Kerndefinitionen des Verfassungsschutzes aus dem Bericht des Jahres 2017, um mögliche Unterschiede in der Konzeptualisierung von Extremismen mit möglicherweise unterschiedlichen Handlungslogiken als Resultat divergierender Konzeptualisierungen herauszuarbeiten.
\end{abstract}

Keywords: Extremismus, öffentlich-medialer Diskurs, Konzeptualisierung, Framing 


\section{Einführung}

Nach zahlreichen terroristischen Gewalttaten in den letzten zwanzig Jahren fällt dem Extremismus wieder ein zentraler Stellenwert in den westlichen Gesellschaften zu. Dabei ist der Extremismus jedoch kein einheitliches Phänomen, sondern durch verschiedene Ausprägungen gekennzeichnet, die sich etwa durch ihre weltanschaulichen Überzeugungen oder ihre Stellung zur Mehrheitsgesellschaft unterscheiden. Die Betonung solcher Aspekte bei der Darstellung der verschiedenen Extremismen in öffentlichen Diskursen kann gesellschaftliche Bewertungen beeinflussen und Argumentationslinien erleichtern oder erschweren (vergleichbar Druckman 2001a, 2001b; Schuck/De Vreese 2006).

Gemeinhin bezeichnet man das Auswählen und Hervorheben von Aspekten eines Sachverhalts, die bestimmte Problemdeutungen und Lösungsansätze befördern, als Framing (Entman 1993: 52). Dabei ist Framing sowohl multiperspektivisch als auch dynamisch: Verschiedene Framings eines Sachverhalts konkurrieren miteinander und sind einem dauerhaften Wandel unterworfen (vgl. Benford/ Snow 2000). Zugleich werden diskursiv entwickelte Framings nicht zufällig ausgewählt, sondern folgen bestimmten Mustern und stehen in Wechselwirkung mit außersprachlichen Entwicklungen. Solche Muster des Framings von gesellschaftlichen Phänomenen und der Wandel dominanter Framings in öffentlichen Diskursen stehen im Fokus der vorliegenden Arbeit. Konkret untersuchen wir, ob und wie Fälle extremistischer Gewalt das Framing von Extremismen im öffentlichen Diskurs beeinflussen. Dabei ist es für unsere Untersuchung unerheblich, ob solche Muster bewusst entwickelt, nachgeahmt, verändert, oder unbewusst übernommen werden.

Um die Untersuchung zu strukturieren, leiten wir zentrale Dimensionen zur Konzeption von Extremismus her. Dabei machen wir uns vornehmlich den Verfassungsschutzbericht zunutze, um vier Kategorien zu entwickeln, die sich zwischen den Extremismusvarianten unterscheiden können und die für das Framing von Extremismus entscheidend sind. Hierbei handelt es sich um: (a) Herkunft der Akteure, (b) Ideologie und Organisation, (c) Stellung zur Gesellschaft und (d) Typische Handlungen. Diese Dimensionen können in der Darstellung des Extremismus unterschiedlich realisiert werden und Wandlungen unterworfen sein. Somit entwickeln wir ein Instrumentarium, um durch externe Ereignisse veränderte Darstellungen des Extremismus abbilden zu können.

Als Untersuchungsfall betrachten wir das Framing des Rechtsextremismus, des Linksextremismus und des Islamismus auf SPIEGEL Online (kurz: SPON) und ob und wie sich die Darstellung der verschiedenen Spielarten des Extremismus nach einschneidenden 
Ereignissen verschoben hat. Durch Betrachtung der Beiträge vor und nach solchen Ereignissen wollen wir herausfinden, ob und wie sie auf das mediale Bild des jeweiligen Extremismus eingewirkt haben.

Methodisch greifen wir auf quantitative Analyseverfahren zurück, um die inhaltliche Ausgestaltung der vier gewählten Dimensionen der Extremismuskonzeption in der Berichterstattung nachzuzeichnen. Dabei untersuchen wir für jede Spielart des Extremismus jeweils ein LE-Feld anstatt von einzelnen lexikalischen Einheiten und setzen uns damit ähnlich Scharloth und Kollegen (2013) das Ziel, das Frame-Konzept (vgl. Abschnitt 2) korpusmethodisch abzubilden. Auf diese Weise kann anhand einer Vielzahl von Instanzen erfasst werden, mit welchen Aspekten die jeweiligen Extremismen assoziiert werden. Damit einher geht, dass wir für die korpusbasierte Analyse einen eher breiten Extremismusbegriff zugrunde legen, der auch mögliche Vorstufen wie Radikalisierung einbezieht, da zum einen zu erwarten ist, dass in der Berichterstattung nicht immer trennscharf zwischen Radikalismus und Extremismus unterschieden wird und zum anderen gerade Übergangs- bzw. Überschneidungsbereiche wichtige Erkenntnisse über die diskursive Vermittlung eines Phänomens bieten können.

Konkret betrachten wir den Anschlag auf das World Trade Center (kurz: 9/11) und die Veröffentlichung des NSU-Skandals (kurz: NSU), die in der deutschen Debatte als die prägendsten Ereignisse im Bereich des Islamismus und im Bereich des Rechtsextremismus gelten dürfen. Eine Reihe von islamistischen Attentaten jüngeren Datums hätten alternativ gewählt werden können - und auch im Bereich des Rechtsextremismus hätte sich mit den Attentaten von Norwegen 2011 ein herausgehobenes Ereignis rechtsterroristischer Prägung als Alternative angeboten. Die Entscheidung für die beiden genannten Fallbeispiele lässt sich vor allem aus zwei Gründen rechtfertigen. Zum einen war das Ziel, die jeweils herausragendsten Ereignisse auszuwählen. Im Bereich des gewaltbereiten Islamismus liegt die Entscheidung für den 11. September 2001 somit auf der Hand. Die Veröffentlichung des NSU-Skandals erschien bedeutsamer für die deutsche Debatte über den Rechtsextremismus als die Attentate in Norwegen. Zweitens sollten Ereignisse ausgewählt werden, die in Zeiten vergleichsweise geringer öffentlicher Aufmerksamkeit für die jeweiligen Extremismen fallen, um einen möglichst unverstellten Blick auf denkbare Verschiebungen in der medialen Darstellung zu erhalten. Schließlich sei noch hinzugefügt, dass der relevante Vergleichszeitpunkt im Bereich des NSU-Skandals die Aufdeckung und nicht etwa der Tatzeitpunkt ist, da die weltanschauliche Dimension der Attentate anfangs nicht erkannt wurde. 
Da es in der jüngeren Vergangenheit im Bereich des Linksextremismus kein Ereignis mit einer ähnlichen Tragweite gibt, analysieren wir die Berichterstattung rund um den G20-Gipfel in Hamburg (kurz: G20), im Rahmen dessen es zu nennenswerten Gewalttaten von linksextremistischer Seite kam. In der deutschen Geschichte gäbe es zweifellos besser geeignete Beispiele, nicht zuletzt Gewalttaten der RAF. Eine Analyse dieser Ereignisse schließt sich jedoch aus Gründen der Datenverfügbarkeit aus.

Zur Entwicklung eines grundlegenden Verständnisses der verschiedenen Extremismen bedienen wir uns zunächst vor allem der Definitionen des Verfassungsschutzes (vgl. Abschnitt 2.2). Zuvor erläutern wir unser Framing-Konzept (Abschnitt 2) und legen anschließend die Korpusdaten sowie die korpusmethodische Operationalisierung dar (Abschnitte 3 und 4). Nachdem wir die Entwicklung der medialen Extremismus-Konzeptionen auf SPON erarbeitet haben (Abschnitte 5.1-5.5), gleichen wir das Vorher-Nachher-Bild (Abschnitt 5.6) mit den Konzeptionen des Verfassungsschutzes ab (Abschnitt 5.7). Damit beleuchten wir zumindest punktuell Divergenzen in der Konzeption von Extremismen, die helfen mögen, Unterschiede zwischen der öffentlich-medialen Darstellung und staatlichen Erklärungs- und Handlungslogiken zu ergründen. Wir thematisieren abschließend (Abschnitt 6) die Stabilität des SPONFramings bezüglich Linksextremismus und Islamismus, sowie die beim Rechtsextremismus zu beobachtende Veränderung im Framing von SPON, das Gewalthandeln erst nach dem NSU-Skandal als Element des Rechtsextremismus aufgreift. Wir diskutieren zudem einen Verzögerungseffekt in der Konzeption des Verfassungsschutzes, der Gewalthandeln und das Agieren im Verborgenen auch 2017 nicht in die Kerndefinition von Rechtextremismus übernimmt.

\section{Frames und Framing}

Die von Robert Entman vertretene Definition von Framing als das Hervorheben bestimmter Teilaspekte eines Themas hat in der Literatur viel Zuspruch gefunden. Dass ein solches Framing einen Effekt auf politische Entscheidungsprozesse und öffentliche Meinung haben kann, ist verschiedentlich nachgewiesen worden, bspw. mit Blick auf den Erfolg der CDU-Wahlkampagne 2013 (Klein 2018), die öffentliche Debatte über Skandale in der jüngeren deutschen Geschichte (Kepplinger et al. 2012), oder aber die mediale Diskussion um die EU-Osterweiterung (Schuck/De Vreese 2006). Was aber ist die kognitive Basis eines Frames und wie kann dieser bei Rezipienten ,funktionieren'? 
Zur Beantwortung dieser Frage knüpft unsere Konzeption des Framing an die Theorie der Frame-Semantik (Fillmore 1982, 1985) an. Die Frame-Semantik ist eine Semantik des Verstehens, die davon ausgeht, dass Menschen zum Verstehen Kategorisierungsschemata in Form systematisch verbundener Konzepte verwenden. Mit Hilfe dieser werden „Szenen“, die wir hier als eine Menge von als zusammenhängend wahrgenommenen Zuständen und Ereignissen definieren ${ }^{1}$, eingeordnet und interpretiert. Ein Frame wird unter anderem durch sprachliche Ausdrücke hervorgerufen (evocation), alle in ihm enthaltenen Konzepte werden dabei aktiviert. Zusätzlich können Rezipienten weitere, für die Interpretation notwendige Frames selbst aktivieren (invocation).

Verschiedene mit einem Frame verknüpfte lexikalische Einheiten geben zugleich eine Perspektive vor. So kann man ein vierbeiniges, bellendes Tier etwa als Hund, als Köter, oder aber als besten Freund des Menschen bezeichnen. Mit jeder Bezeichnung wird eine Wertung transportiert, von neutral über abwertend bis hin zu aufwertend. ${ }^{2}$ Auch lässt sich auf diese Weise steuern, welche Aspekte in den Vordergrund gerückt werden: Während der beste Freund des Menschen eher die Rolle als Haustier betont, streichen Köter oder Vierbeiner das Tiersein hervor. Über die Benennungswahl können wir also eine Perspektive auf einen Erfahrungsbereich vorbestimmen und die Interpretation leiten.

Neben einer unterschiedlichen Perspektivierung durch die $e v o-$ cation steuert auch die invocation, also der Abruf von Hintergrundinformation zu einem Konzept, die Interpretation eines Begriffs. Ausdrücke werden somit nicht nur über die durch sie aufgerufenen frame-internen Konzepte, sondern ebenfalls über frame-externe Konzepte interpretiert. So ist mit einigen Tierarten beispielsweise das Konzept Gefahr verbunden, was in spezifischen Verhaltensweisen resultieren sollte. Dabei können sich die Interpretationen aber durchaus unterscheiden. So mag die Wahrnehmung des Gefahrenpotenzials für Hund recht verschieden sein, die Abrufmuster von Frames sind folglich in gewissem Grade rezipientenspezifisch, ob individuell oder gruppenbezogen. Wir gehen hier davon aus, dass die Abrufmuster (patterns of invocation) über das Framing, d.h. über die Einbettung eines Frames in weitere Frames, in einer konkreten Äußerung sowohl im Einzelfall als auch durch das wiederholte Framing beeinflusst werden können. Gehäufte Berichte der Art „Erneute Hundeattacke“ würden demnach eine Verknüpfung

1 Zur Problematik von außersprachlichen Realitäten vgl. Jäger (2018).

2 Für dieses und weitere Beispiele, siehe https://derzwiebel.wordpress.com/ 2019/10/08/gefluechtet-oder-geflohen-welches-verb-wird-wann-haeufigergenutzt/, abgerufen am 18. Oktober 2019. 
zwischen Hund und Gefahr befördern, anders wäre dies bei einer öffentlichen Debatte über die Steuerpflicht für die Hundehaltung.

Im vorliegenden Beitrag untersuchen wir Abrufmuster und modellieren dies durch die Analyse von Assoziationen von Ausdrücken, also über das gemeinsame Auftreten bestimmter Ausdrücke (vgl. Abschnitt 4.2). Durch die Auswahl und Belegung verschiedener Aspekte eines Sachverhalts können, so die Annahme, Interpretationen und daraus folgende Handlungsalternativen entscheidend beeinflusst werden.

\subsection{Konzeptualisierung und Framing von Extremismus}

Die vergleichende Untersuchung der sprachlichen Konzeptualisierung und des Framings der unterschiedlichen Varianten des Extremismus stellt ein bisher nur wenig bearbeitetes Forschungsfeld dar. Studien über den Zusammenhang von Extremismus und Sprache konzentrieren sich vor allem auf zwei Aspekte:

- die Sprache von Extremisten, beginnend mit Viktor Klemperers 1947 erstmals erschienenem LTI. Notizbuch eines Philologen (2018) und in verschiedenen neueren Arbeiten (z. B. Schuppener 2010; Ebling et al. 2014; Liebert 2019)

- die Abgrenzung des Konzepts Extremismus von benachbarten Konzepten wie Radikalismus oder Terror (Bötticher/Mareš 2012; Ackermann et al. 2015; Bötticher 2017)

Unterschiede und ein möglicher Wandel des Verständnisses der verschiedenen Ausprägungen von Extremismus werden dagegen zwar vereinzelt aufgegriffen, aber nicht systematisch ausgearbeitet. So untersucht Bötticher (2017) beispielsweise die Konzeptualisierungen von Radikalismus und Extremismus im fachsprachlichen Extremismusdiskurs des deutschsprachigen Raums. Dabei fasst sie den Extremismus als Oberbegriff ins Auge, die einzelnen Ausprägungen des Extremismus werden hauptsächlich im Hinblick auf mögliche gemeinsame Merkmale diskutiert. Ackermann und Kollegen (2015) beschäftigen sich mit einem umfassenden Extremismusbegriff, gehen aber zumindest punktuell auf unterschiedliche Entwicklungen der Begriffe Linksextremismus und Rechtsextremismus ein.

Auch Studien zu einzelnen Ausprägungen des Extremismus stellen eher die Ausnahme dar. So untersucht etwa Lasch (2014), wie die Begriffe Islamismus und Islamisten sich von der Bezeichnung für eine Religionsgemeinschaft zu Schlagwörtern für eine religiös motivierte, gewaltbereite Bewegung entwickelt haben. 
Entscheidend für das Verständnis von Extremismus ist jedoch nicht nur die Konzeptualisierung des Grundbegriffs, sondern ebenso die Konzeptualisierung seiner unterschiedlichen Ausprägungsformen, die Einbettung dieser Konzepte in Deutungszusammenhänge und ihre Veränderung über Zeit. Die Untersuchung der Konzeptualisierungen und des Framings der verschiedenen Extremismen ist besonders deshalb geboten, da sie bestimmte Argumentationslinien und Handlungslogiken sprachlich hervorheben, andere dagegen verneinen und auf diese Weise angemessenes politisches Handeln vorstrukturieren und zugleich gesellschaftlichen Meinungsbildungen Vorschub leisten.

\subsection{Konzeptuelle Dimensionen des Extremismus}

Zur Erarbeitung der relevanten Dimensionen für die Konzeption von Extremismen ziehen wir die in Kooperation mit dem Bundeskriminalamt entstandene vergleichende Untersuchung über das Gewalthandeln extremistischer Strömungen von Mletzko (2010) und die Definitionen verschiedener Extremismen aus dem Verfassungsschutzbericht des Jahres 2017 heran (Bundesministerium des Innern, für Bau und Heimat 2017).

Mletzko (2010) untersucht das Gewalthandeln militanter linker und rechter Szenen und stellt fest:

Zunächst gilt es, die Kernszenen, aus denen heraus Gewalttaten verübt werden, mit einigen Stichworten zu beschreiben. Im linken Feld spielt die aus dem Sponti-Milieu der 1970er Jahre hervorgegangene, seit Anfang der 1980er Jahre existierende politische Szene autonomer Gruppen eine Schlüsselrolle. [...] Nicht minder heterogen stellt sich der Phänomenbereich des gewaltbereiten Rechtsextremismus dar, dem verschiedene Teilspektren zuzurechnen sind: die im Wandel begriffene Szene der Neonationalsozialisten und die Rechtsrockszene sowie weltanschaulich und stilistisch verwandte Hooligan- und Rockermilieus. (Mletzko 2010: 10-11)

Mletzko benennt also die Quellen rechtsextremer und linksextremer Szenen, was wir im Folgenden unter der Dimension Herkunft der Akteure fassen. Weiter widmet sich Mletzko dem Gewalthandeln der verschiedenen Szenen mit feinerer Untergliederung: Er charakterisiert rechtsextreme Gewalt als stärker lebensbedrohlich und gegen Menschen mit anderen ethnischen oder religiösen Hintergründen gerichtet, linksextreme Gewalt als seltener lebensbedrohlich und vor allem gegen das rechtsextreme Milieu und die 
Staatsgewalt gerichtet. Die hier berührte Dimension fassen wir mit dem Begriff Typische Handlungen.

Im Verfassungsschutzbericht wird die Einteilung des Extremismus in drei große Kategorien deutlich: Rechtsextremismus, Linksextremismus und Islamismus (gelegentlich auch Salafismus). Der Verfassungsschutz fokussiert in seiner Charakterisierung der unterschiedlichen Extremismen zunächst auf weltanschauliche Elemente.

Prägender Teil dessen, was wir im Folgenden als Ideologie bezeichnen, ist beim Rechtsextremismus ein „,völkisches“ Verständnis von Gesellschaft, welches im Gegensatz zu zentralen Werten des Grundgesetzes und der freiheitlich-demokratischen Grundordnung steht, aber nicht notwendigerweise zum Staat an sich, da „Nation“ Element der Gruppenidentifikation ist:

Die Zugehörigkeit zu einer Ethnie, Nation oder Rasse ist im rechtsextremistischen Weltbild entscheidend für den Wert eines Menschen. Diesem „völkischen“ Kriterium sind auch die Bürger- und Menschenrechte des Einzelnen untergeordnet. Rechtsextremistische Agitation ist geprägt von Fremdenfeindlichkeit und Rassismus, Antisemitismus und Geschichtsrevisionismus sowie einer grundsätzlichen Demokratiefeindschaft. Damit stehen Rechtsextremisten und deren Ideologie im fundamentalen Widerspruch zu zentralen und universellen Werten des Grundgesetzes, das die Würde des Menschen in den Mittelpunkt stellt. (Bundesministerium des Innern, für Bau und Heimat 2017: 44)

Mit Blick auf die Herkunft der Akteure stellt der Verfassungsschutz somit fest, dass der Rechtsextremismus ein aus der Gesellschaft entspringender Extremismus ist. Dieser richtet sich vor allem gegen Menschen, die als außenstehend bzw. von außen kommend oder von einer empfundenen Norm abweichend angesehen werden. Dieser Extremismus ist nicht gegen die eigene Gesellschaft gerichtet, sondern zielt auf eine „Reinhaltung“ der Gesellschaft ab. Dies bezeichnen wir als die Dimension Stellung zur Gesellschaft. Typische Handlungen sind in dieser Definition nicht abgebildet.

Der Linksextremismus wird vom Verfassungsschutz wie folgt charakterisiert:

Linksextremisten verfolgen das Ziel, unsere Staats- und Gesellschaftsordnung und damit die freiheitliche Demokratie abzuschaffen und durch ein kommunistisches oder ein „herrschaftsfreies“, anarchistisches System zu ersetzen. Ihre theoretischen Leitfiguren sind - in unterschiedlichem Ausmaß 
und abweichender Interpretation - Marx, Engels und Lenin. Gewalt, verstanden als „revolutionäre Gewalt“ der „Unterdrückten gegen die Herrschenden“, gilt grundsätzlich als legitim. (Bundesministerium des Innern, für Bau und Heimat 2017: 100)

Der Linksextremismus steht also weniger der Gesellschaftsform, sondern eher der darin verankerten Herrschaftsordnung entgegen. Ihre ideologischen Überzeugungen werden hier durch Nennung entscheidender Figuren ebenso explizit gemacht wie auch Gewalt als Typische Handlung klar benannt wird. Die Herkunft der Akteure des Linksextremismus bleibt in dieser Charakterisierung ungenannt.

Der Islamismus ist laut Definition des Verfassungsschutzes religiös motiviert und steht grundlegenden Prinzipien des Staates entgegen, etwa der Trennung von Staat und Religion und der Volkssouveränität:

Der Begriff „Islamismus“ bezeichnet eine Form des politischen Extremismus. Unter Berufung auf den Islam zielt der Islamismus auf die teilweise oder vollständige Abschaffung der freiheitlichen demokratischen Grundordnung der Bundesrepublik Deutschland ab. Der Islamismus basiert auf der Überzeugung, dass der Islam nicht nur eine persönliche, private „Angelegenheit" ist, sondern auch das gesellschaftliche Leben und die politische Ordnung bestimmt oder zumindest teilweise regelt. Der Islamismus postuliert die Existenz einer gottgewollten und daher „wahren“ und absoluten Ordnung, die über den von Menschen gemachten Ordnungen steht. Mit ihrer Auslegung des Islam stehen Islamisten insbesondere im Widerspruch zu den im Grundgesetz verankerten Grundsätzen der Volkssouveränität, der Trennung von Staat und Religion, der freien Meinungsäußerung und der allgemeinen Gleichberechtigung. Ein wesentliches ideologisches Element des Islamismus ist außerdem der Antisemitismus. (Bundesministerium des Innern, für Bau und Heimat 2017: 164)

In der Beschreibung werden die Akteure in verschiedenen, mehr oder weniger gewaltbereiten Gruppen (Gewalt und Terrorismus als Typische Handlung) verortet, die sich aber nicht klar lokalisieren lassen (diffuse Herkunft der Akteure). Der Islamismus will laut dieser Definition die aktuelle Gesellschaft auf Basis einer strengen und allgültigen Auslegung religiöser Inhalte grundlegend verändern, wobei antisemitische Haltungen ebenfalls als Teil der Ideologie des Islamismus verstanden werden. 
Im Folgenden analysieren wir die Berichterstattung zu den Extremismen im Vor- und Nachgang extremistischer Gewaltereignisse mit Blick auf das assoziative Framing der Extremismen. Dabei greifen wir auf die vier in diesem Abschnitt herausgearbeiteten Dimensionen Herkunft der Akteure, Stellung zur Gesellschaft, Typische Handlungen und Ideologie zurück. Letzteres erweitern wir um den Aspekt der Organisation. Organisationsfragen sind für die grundlegenden Definitionen zwar nicht wesentlich, spielen in der medialen Diskussion aber eine zentrale Rolle und sollten entsprechend berücksichtigt werden. Im Anschluss prüfen wir, inwiefern sich die hier ausgeführten Definitionen des Verfassungsschutzes mit den im medialen Diskurs zu beobachtenden Perspektiven decken, widersprechen oder komplementieren.

\section{Korpusdaten}

\subsection{SPIEGEL Online}

Wir untersuchen das Framing von Extremismen auf der OnlineNachrichtenplattform SPON vor und nach sichtbaren extremistischen Gewaltereignissen. Diese Entscheidung ist vor allem durch den für eine webzentrierte Studie ausgesprochen langen Untersuchungszeitraum zu begründen, der bis Januar 2000 zurückreicht und somit einen Zeitraum von fast zwanzig Jahren umfasst. Hier bietet sich Spiegel Online an, da sich die Seite früh als Online-Nachrichtenportal im deutschen Markt hervorgetan hat (Meyer-Lucht 2007) und seitdem zu den einflussreichsten und meistgesehenen Portalen gehört $^{3}$, dem unter den Online-Medien eine Meinungsführerschaft zufällt (Bönisch 2006). Mit der Analyse von SPON kann somit zweierlei gewährleistet werden. Erstens wird die Untersuchung auf Grundlage eines besonders einflussreichen Mediums in der deutschen Medienlandschaft durchgeführt, sodass Zuschreibungen von Bedeutungszusammenhängen und deren Wandel das Meinungsklima entscheidend prägen können. Zum anderen wird die Vergleichbarkeit zwischen den drei Fallbeispielen sichergestellt, indem die Berichterstattung im gleichen Medium zugrunde gelegt wird.

Die Auswahl eines Online-Mediums im Speziellen ist einerseits forschungspraktischen Erwägungen geschuldet. So liegt das gesamte Nachrichtenarchiv von SPON in digitalisierter und maschinenlesbarer Form frei zugänglich vor, was die Datensammlung und -analyse erheblich erleichtert. Andererseits kann mit der Analyse eines

3 https://www.agof.de/studien/internet-facts/studienarchiv-internet/, abgerufen am 8. Dezember 2019. 
Online-Mediums dem zuweilen vorgetragenen Vorwurf begegnet werden, dass Auswertungen von Printmedien sich mit einem Gegenstand mit zunehmend geringerer Breitenwirkung beschäftigen - ein Vorwurf, der freilich einen keineswegs zwingenden Unterschied in der Berichterstattung zwischen Online- und Offline-Medien voraussetzt (Ghersetti 2014; Vonbun-Feldbauer/Matthes 2018). Die Auswahl von SPON im Gegensatz zu vergleichbaren Nachrichtenportalen wie bild.de oder Focus Online begründet sich schließlich neben der Bedeutung und Langlebigkeit der gewählten Plattform auch durch die vergleichsweise moderate und wenig offensiv vorgetragene weltanschauliche Positionierung von SPON.

Trotz der zahlreichen Argumente, die sich für die Auswahl von Spiegel Online anführen lassen, ist bei der Auswertung selbstverständlich zu berücksichtigen, dass wir lediglich einen Ausschnitt der deutschen Medienlandschaft betrachten. Während es sich bei SPON also durchaus um ein Leitmedium mit entsprechender Breitenwirkung handelt, so können dennoch kaum Rückschlüsse aus der Analyse über die Darstellung von Extremismus in der weiteren deutschen Medienlandschaft gezogen werden.

\subsection{Korpuserstellung}

Die Korpusdaten bestehen aus Artikeln auf SPON, aus einer Phase von rund einem Jahr vor und nach dem gewählten Ereignis. Die Texte wurden automatisiert heruntergeladen und die Artikeltexte ohne Kommentarspalten oder anderes Material extrahiert. Versehen wurden die resultierenden Texte mit dem Ursprungslink sowie Jahr, Monat und Tag der Veröffentlichung als Metadaten, wobei die letzte Veröffentlichungsversion nach möglichen Redaktionskorrekturen ausschlaggebend war.

Im Ergebnis erhalten wir sechs Subkorpora: pre911 und post911 für die Phasen vor und nach dem Anschlag auf das World Trade Center, analog dazu preNSU und postNSU sowie preG20 und postG20. Die Zeiträume sind nicht exakt ausgeglichen, die Subkorpusgrößen und Zeiträume bewegen sich jedoch in einem jeweils vergleichbaren Bereich. 


\begin{tabular}{|l|l|l|}
\hline Subkorpus & Zeitraum & \# Wörter \\
\hline pre911 & $2000-01-01-2001-09-10$ & 1.956 .760 \\
\hline post911 & $2001-09-11-2002-12-31$ & 1.581 .967 \\
\hline preNSU & $2011-01-01-2011-11-03$ & 1.294 .925 \\
\hline postNSU & $2011-11-04-2012-12-31$ & 1.933 .906 \\
\hline preG20 & $2016-07-01-2017-07-06$ & 1.447 .926 \\
\hline postG20 & $2017-07-07-2018-06-30$ & 1.619 .790 \\
\hline
\end{tabular}

Tabelle 1: Auswahlzeitraum und Größe der Subkorpora

\section{Strategien zur Quantifizierung des medialen Framings}

\subsection{Abgrenzung von LE-Feldern}

Für die quantitative Textanalyse ist es notwendig, dass das untersuchte Thema in genügend Texten bzw. Textabschnitten sprachlich realisiert ist. Dabei ist es möglich, über ein Thema nicht nur mit Hilfe der konkret bei einer Korpusanalyse zuzuordnenden Suchwörter zu sprechen, sondern ebenso anaphorisch, kataphorisch oder in metaphorischer Weise (d. h. auch auf eine Art, die nicht in den Suchwörtern wiedergespiegelt ist). Solche Fälle klammern wir in unserer Studie in der Erwartung aus, dass eine gut abgesteckte Menge von Suchwörtern eine repräsentative und aufschlussreiche Zahl von Fundstellen sicherstellt.

Die Identifikation der für eine Untersuchung relevanten Texte oder Textabschnitte steht und fällt also mit der gelungenen Definition von Suchwörtern. Um ein thematisch breites Feld wie Extremismus abzudecken, genügt eine eng gefasste Menge von Suchwörtern (etwa nur Rechtsextremismus und rechtsextremistisch) aus unserer Sicht nicht; das Bild bliebe unvollständig. Diesem Umstand begegnen wir, indem wir LE-Felder, d. h. Felder lexikalischer Einheiten eingrenzen, die für die Darstellung der jeweiligen Extremismen konstitutiv sind.

Die von uns genutzten Suchwörter bezeichnen wir nach framesemantischer Tradition als lexikalische Einheiten, weil wir davon ausgehen, dass sie bei Rezipienten entsprechende Frames evozieren. Auf eine Menge von lexikalischen Einheiten beziehen wir uns als LE-Felder, bei deren Abgrenzung wir neben der Ausnutzung von 
semantischen Relationen wie etwa Synonymie auch andere konzeptuelle Erwägungen wie z. B. typische Akteure miteinbeziehen.

Die Idee, LE-Felder und nicht einzelne lexikalische Einheiten für die Analyse heranzuziehen, basiert auf dem in Abschnitt 2 erläuterten Frame-Konzept. Sprachlich kann ein Frame durch unterschiedliche lexikalische Einheiten realisiert werden. Durch eine quantitative Auswertung auf Grundlage von LE-Feldern wollen wir möglichst viele Instanziierungen des Frames abdecken.

In Erweiterung der Arbeit von Scharloth und Kollegen (2013) bedienen wir uns nicht nur eines Thesaurus, also einer gegebenen Wortliste für ein Themengebiet, sondern extrahieren weitere lexikalische Einheiten mittels eines Ankers aus einem Korpus. Dabei werden Nomina wie Adjektive berücksichtigt. Die aus dem Korpus extrahierten lexikalischen Einheiten dienen als Grundlage für die LE-Felder, die durch einen menschlichen Leser geprüft und nötigenfalls überarbeitet werden (s. u.). Die Wortprofile für NSU bzw. 9/11 wurden Stand Januar 2018 erstellt, für G20 Stand August 2018.

Um die für den Rechtsextremismus, den Linksextremismus und den Islamismus relevanten LE-Felder abzustecken, wurden die Anker rechtsextremistisch, linksextremistisch und islamistisch herangezogen. Die resultierenden LE-Felder benennen wir im Folgenden $\mathrm{RE}$ aus dem Anker rechtsextremistisch, LI aus dem Anker linksextremistisch und IM aus dem Anker islamistisch. Um die LE-Felder möglichst breit aufzustellen, wurde das umfangreiche Kernkorpus des Digitalen Wörterbuchs der Deutschen Sprache (kurz: DWDS; Klein/Geyken 2010) als Extraktionsbasis genutzt. Zwei Funktionen des DWDS dienen dabei zur Generierung von LE-Feldkandidaten.

Zum einen waren die im OpenThesaurus gelisteten Synonyme natürliche Kandidaten. Daraus resultierten weitere adjektivische Beschreibungen für das LE-Feld wie etwa rechtsradikal oder faschistisch, wie in Abbildung 1 dargestellt.

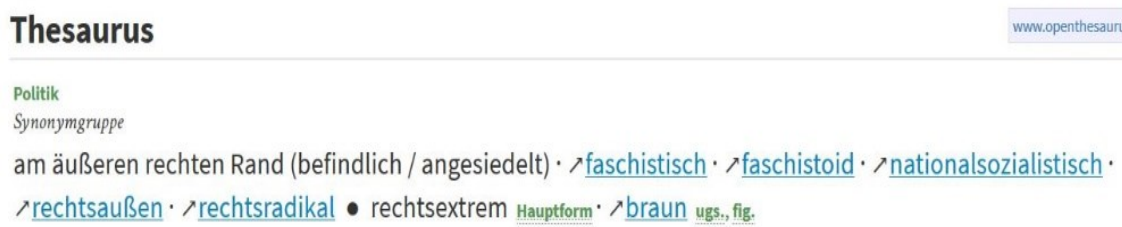

Abbildung 1: Thesauruseintrag für rechtsextremistisch im DWDS (Oktober 2017)

Zum anderen nutzten wir die DWDS-Funktion ,Wortprofil'. Die Wortprofilfunktion berechnet typische Wortverbindungen, die in verschiedenen grammatischen Beziehungen zum Suchwort stehen (Didakowski/Geyken 2014). 


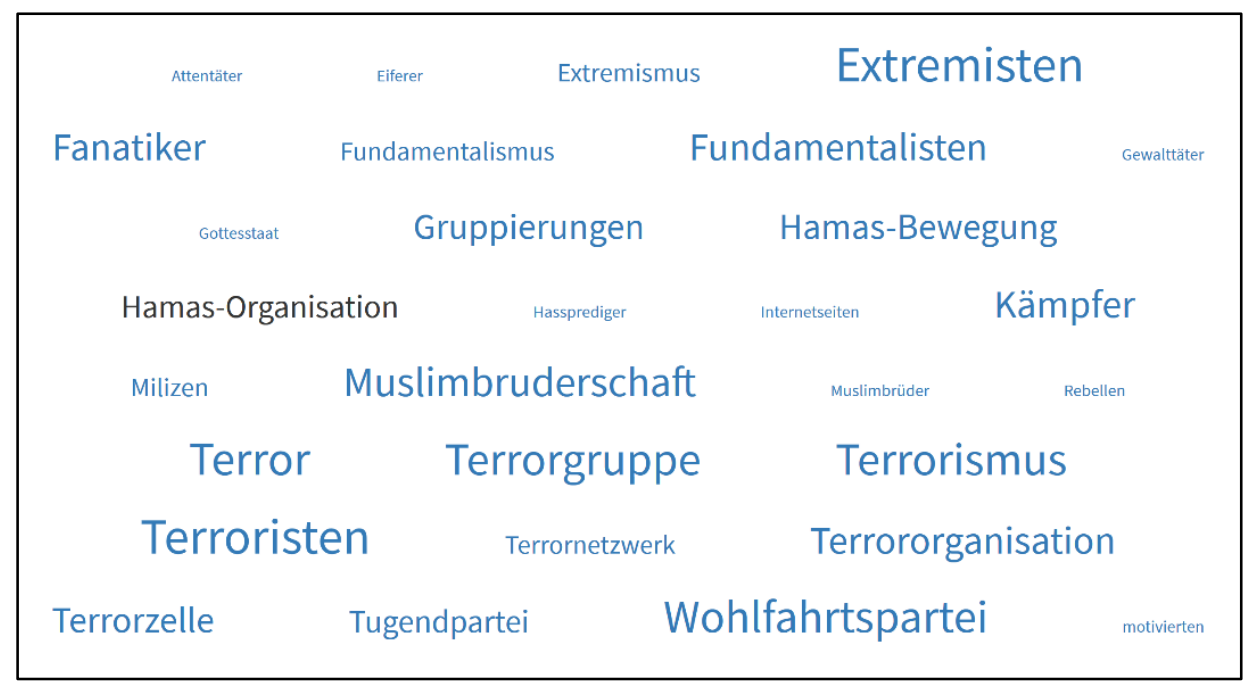

Abbildung 2: Grafische Darstellung der Top-Treffer des Wortprofils zu islamistisch im DWDS

Die Wortprofile der Anker waren informativ, aber interpretationsbedürftig. Die Wortwolke in Abbildung 2 stellt einen Ausschnitt der häufigsten Kollokate aus verschiedenen Kategorien (z.B. Adjektivattribut von, Adverbialbestimmung von) für das Suchwort rechtsextremistisch dar. Eine Reihe von Aspekten können zwar mit dem LE-Feld rechtsextremistisch assoziiert sein, wären aber einem jeweils eigenen Phänomenbereich bzw. Frame zuzuordnen, zum Beispiel der gesamte Terrorbereich. Solche lexikalischen Einheiten werden verworfen. Aus dem Wortprofil konnten aber bspw. relevante Eigennamen übernommen werden, etwa $N P D$ (Bereich RE) oder Hamas (Bereich IM). Zusätzlich ergänzten wir LE-Felder auf Grundlage unseres domänenspezifischen Wissens. Dies bezog sich insbesondere auf relevante Akteure, etwa die Gruppierung AlQaida, die im DWDS-Wortprofil bei Standardeinstellung (max. 20 Kollokate pro Kategorie) nicht auftrat. Die resultierenden LE-Felder sind im Anhang gelistet.

\subsection{Assoziationen mit den LE-Feldern}

Die LE-Felder RE, LI und IM dienen als Ausgangspunkt für die Analyse der vier Konzeptionsdimensionen von Extremismus in den SPON-Daten. Das Framing der Extremismusvarianten lesen wir an unterschiedlichen Assoziationen mit den LE-Feldern, die einer der vier Dimensionen zuzuordnen sind, ab. Die sprachlich konkretisierten Assoziationen sehen wir hier als Ausnutzung des Prinzips des patterns of invocation: Werden beispielsweise Gewaltbereitschaft und eine Extremismusform häufig gemeinsam verhandelt, sollte dies die Invokationsverbindung dieser beiden Frames stärken. 
Zur Ermittlung der Assoziationen verwenden wir die WordSketch-Funktion (Baisa 2010) des Korpuswerkzeugs SketchEngine (Kilgarriff et al. 2014). Diese ist vergleichbar mit der WortprofilFunktion des DWDS: Kollokate werden nach ihrer grammatischen Position zum gesuchten Wort klassifiziert, also beispielsweise typische Subjekte eines Verbs oder die von einem Adjektiv üblicherweise modifizierten Nomen. Wir leiten aus diesen Beziehungen ab, dass die Kollokate in einem Sinnzusammenhang mit den jeweiligen Suchwörtern stehen. Mit dieser methodischen Entscheidung fokussieren wir uns auf den Satzkontext und schließen andere denkbare Operationalisierungen von Assoziation aus.

In Abbildung 3 ist ein Auszug der für das LE-Feld RE kumulierten Daten in der Phase nach der Veröffentlichung des NSU-Skandals dargestellt; die vollständigen Häufigkeitsangaben finden sich im Anhang $^{4}$ zu diesem Beitrag. Zur einfacheren Übersicht sind die Informationen in den Abbildungen 4-9 als Wortwolken dargestellt. Kollokate werden nach dem grammatischen Bezug zum Suchwort geordnet, so bezieht sich beispielsweise die hier dargestellte Kategorie nouns modified by lemma nur auf adjektivische Suchwörter und die von ihnen modifizierten Nomen.

\begin{tabular}{l|c|l}
\hline lemma & pos & nouns modified by lemma \\
\hline NSU (167) & $-n$ & \\
\hline Hooligan (10) & $-n$ & \\
\hline NPD (524) & $-n$ & \\
\hline rechtsextrem (368) & $-j$ & $n p d-n(40)$, szene-n (33), terrorzelle-n (12), partei-r \\
\hline Rechtsextreme (113) & $-n$ & \\
\hline Rechtsextrem (3) & $-n$ & \\
\hline fremdenfeindlich (15) & $-j$ & ausschreitung-n (2), ressentiment-n (1), übergriff-r \\
\hline rechtsradikal (42) & $-j$ & trio-n (2), gedankengut-n (3), nsu-zelle-n (1), mörd \\
\hline antisemitisch (65) & $-j$ & hetze-n (2), klischee-n (2), hass-mail-n (1), weltsic \\
\hline braun (73) & $-j$ & sumpf-n (5), terror-n (6), grauen-n (3), brut-n (2), s
\end{tabular}

Abbildung 3: Auszug: lexikalische Einheiten mit Häufigkeit (linke Spalte) und syntaktisch in Beziehung stehende Kookkurrenzen mit Häufigkeit (rechte Spalte) aus dem Subkorpus postNSU

Da die WordSketch-Funktion nur auf Grundlage von einzelnen Suchwörtern operiert, wurde auf die SketchEngine-API zurückgegriffen, um den WordSketch für jede lexikalische Einheit eines LEFelds zu errechnen und die Ergebnisse dann für ein LE-Feld zu kumulieren. Aufgenommen wurden nur Kollokate, die mindestens zweimal auftraten. Die grammatischen Kategorien wurden für die weitere Analyse nicht berücksichtigt.

4 Abzurufen von der Seite des Beitrags unter https://www.jfml.org. 


\section{Analyse}

Bei der Analyse berücksichtigen wir zwei Aspekte der Korpusergebnisse. Zum einen betrachten wir die Frequenz der Auftreten von Instanzen aus den LE-Feldern, was zwar eher dem Bereich AgendaSetting als dem Framing zuzuordnen wäre, an dieser Stelle aber nicht ignoriert werden kann. In Tabelle 2 ist die Anzahl der lexikalischen Einheiten und der Kollokate jeweils mit der Gesamtzahl ihrer Instanzen aufgeführt. Die relative Frequenz berechnen wir wie folgt:

relFreq = Gesamtzahl (Instanzen) $* 1.000$ / Gesamtzahl (Wörter im Subkorpus)

Zum anderen interpretieren wir die mit den LE-Feldern assoziierten Kollokate mit Blick auf die vier Dimensionen Herkunft der Akteure, Stellung zur Gesellschaft, Ideologie und Organisation und Typische Handlungen. Obwohl sich diese Dimensionen als hilfreich und sinnvoll für die Strukturierung der Beobachtungen erweisen, muss dennoch bemerkt werden, dass einzelne lexikalische Einheiten sich an der Grenze von mehreren Dimensionen bewegen können und dies in der Diskussion entsprechend zu berücksichtigen ist. Anschließend untersuchen wir exemplarisch, inwiefern sich die in den Korpusdaten zu beobachtenden Muster mit den Definitionen des Verfassungschutzes der Extremismen decken.

\subsection{Frequenz}

Die in den Abbildungen 4-9 dargestellten Kollokate der LE-Felder stellen Befunde vor und nach dem untersuchten Ereignis dar. Für eine lesbare Darstellung wurden jeweils unterschiedliche Werte für die Mindesthäufigkeit eines Kollokats gewählt (in den Abbildungsunterschriften in Klammern angegeben).

Die Darstellung in Form von Wortwolken ist nicht optimal, allerdings ist uns keine Darstellung bekannt, welche sich für die Beziehung zwischen zwei Mengen von Wörtern (hier: LE-Feld und Kollokate) eignen würde. 


\begin{tabular}{|c|c|c|c|c|c|c|}
\hline Subkorpus & 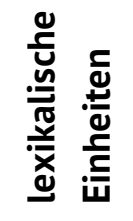 & 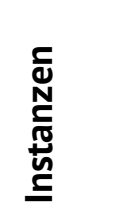 & 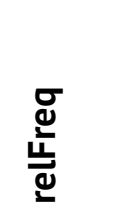 & $\begin{array}{l}\stackrel{0}{0} \\
\stackrel{0}{0} \\
\stackrel{0}{0} \\
\stackrel{0}{0} \\
\stackrel{1}{0}\end{array}$ & 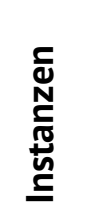 & 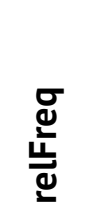 \\
\hline pre911 & 7 & 15 & 0,007 & 14 & 15 & 0,007 \\
\hline post911 & 9 & 313 & 0,198 & 141 & 217 & 0,137 \\
\hline preNSU & 22 & 636 & 0,491 & 225 & 374 & 0,289 \\
\hline postNSU & 26 & 1711 & 0,885 & 375 & 998 & 0,516 \\
\hline preG20 & 8 & 62 & 0,042 & 35 & 49 & 0,034 \\
\hline postG20 & 7 & 171 & 0,106 & 93 & 126 & 0,078 \\
\hline
\end{tabular}

Tabelle 2: absolute und relative Anzahl von lexikalischen Einheiten und Kollokaten sowie deren Instanzen

Aus Tabelle 2 können wir mit Blick auf die Frequenz des Auftretens von LE-Feldinstanzen folgende Schlüsse ziehen. Wurde vor dem NSU-Skandal der Rechtsextremismus bereits ausgiebig diskutiert, so geschah dies nach der Aufdeckung des Skandals mit einer etwa doppelt so hohen Frequenz. Im Falle des 11. September ist der Anstieg mit einem Faktor von etwa 20 als massiv zu bezeichnen. Im Falle des LE-Felds LI ist ebenfalls eine Steigerung für die Zeit nach G20 im Vergleich zum Zeitraum vor G20 zu verzeichnen. Allerdings fällt die Steigerung mit einem Faktor von zwei bis drei nicht annähernd so deutlich aus wie im Falle des Islamismus. Dies mag sich auch dadurch erklären, dass schon im Vorfeld von G20 mit linksextremistischen Übergriffen gerechnet wurde und die Berichterstattung deshalb bereits zunahm. Für die Erfassung von Frequenzen außerhalb dieses Ereignisses wäre eine kontinuierliche Abbildung des Thementrends somit geeigneter, die Auswertung der Frequenz steht in diesem Beitrag aber nicht im Vordergrund.

Im Folgenden widmen wir uns den Inhalten der Berichterstattung und wie diese sich nach den gewählten Ereignissen verschoben hat. Hintergrund dieser Betrachtung ist die Überlegung, dass die Häufigkeit der LE-Feldinstanzen zwar etwas über den Stellenwert eines Phänomens in der öffentlich-medialen Darstellung aussagt, nicht aber über die damit verknüpften Konzeptionen und Handlungslogiken. 


\section{pre911: Collocates}

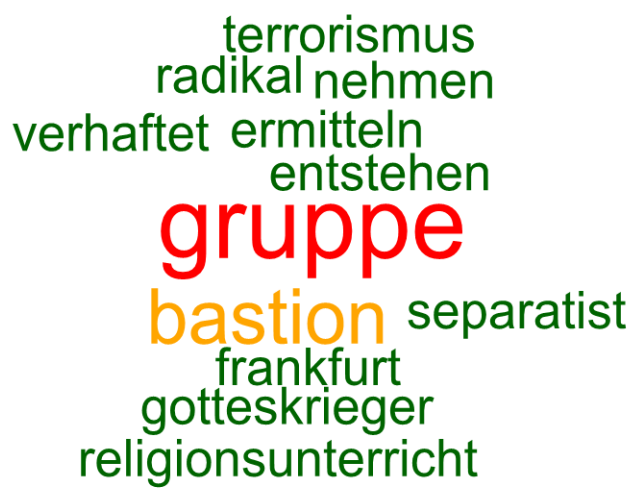

Abbildung 4: Kollokate für das LE-Feld IM vor den Anschlägen vom 11. September 2011 (Mindestfrequenz: 1)

\section{post911: Collocates}

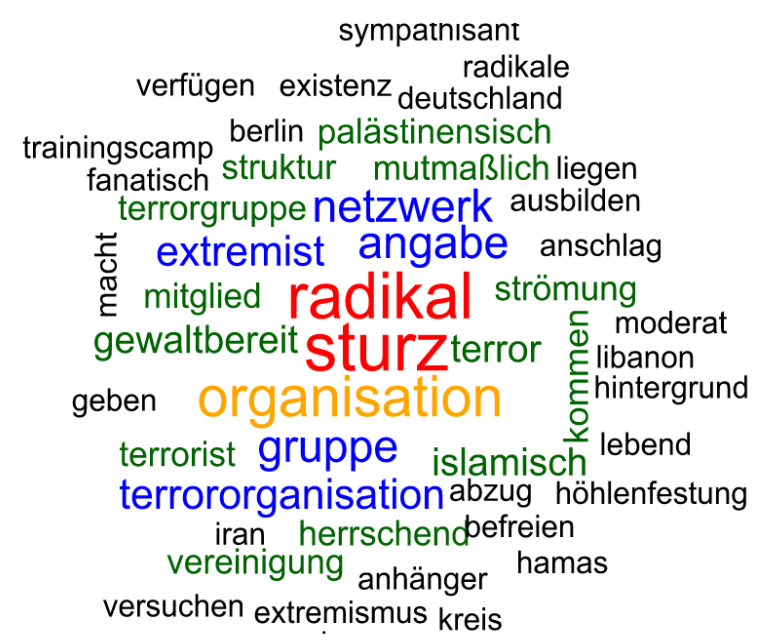

Abbildung 5: Kollokate für das LE-Feld IM nach den Anschlägen vom 11. September 2001 (Mindestfrequenz: 2)

\subsection{Dimension: Herkunft der Akteure}

In den Kollokaten für IM aus post911 wird die Lokalisierung (scheinbar) relevanter Akteure konkretisiert (Iran, Libanon) und Akteure benannt (Hamas). Die Daten aus pre911 benennen keinen Ort und keine konkrete Gruppierung außerhalb des deutschen Staatsgebiets. In beiden Fällen wird aber ein in Deutschland gelegener Ort (Frankfurt bzw. Berlin) thematisiert. Mit Blick darauf, dass Islamismus sich in post911 als externe Bedrohung darstellt, könnte man diese Orte als Einfallstore auffassen, wie in den folgenden Satzbeispielen: 
(1) Gegen die vier in Frankfurt inhaftierten Islamisten [...] (preNSU)

(2) [...] von in Berlin ansässigen Sympathisanten radikaler Islamisten... (postNSU)

Rechtsextremismus wird in preNSU wie postNSU als Gefahr von innen dargestellt. Dabei erscheint Rechtsextremismus als Phänomen, das in der Mitte der Gesellschaft vorkommt (Gedankengut, Tendenz), sich aber durchaus in eigenen Gruppierungen sammelt (Szene, Milieu). Entsprechende „Tendenzen“ werden zum Beispiel auch an Hochschulen vorgefunden:

(3) Das Ansehen der Hochschule ist jetzt durch die rechtsextremen Tendenzen in der Studentenzeitung beschädigt worden. (preNSU)

In einem Beitrag über die Piratenpartei wird das Thema möglicher rechtsextremer Tendenzen ebenfalls aufgegriffen:

(4) Nerz wies den Vorwurf zurück, es gebe rechtsextreme Tendenzen in der Partei: (postNSU)

In postNSU taucht mit Untergrund ein zuvor nicht aufgeführtes Kollokat auf, das für rechtsextreme Aktivitäten einen neuen, zuvor unbeachteten Ort benennt. Hierbei handelt es sich um ein echtes Kollokat, jedes Vorkommen von „Untergrund“ als Teil der ausgeschriebenen Variante von $N S U$ wurde explizit aus der Treffermenge herausgenommen. Die Selbstbeschreibung der Organisation NSU als im Untergrund agierende Gruppierung wurde also von SPON übernommen. Dies erweitert die Dimension Herkunft der Akteure um einen vor November 2011 nicht mit dem Rechtsextremismus assoziierten Aspekt.

Linksextremismus wird sowohl in preG20 als auch in postG20 als entfernter, abgesonderter Teil der Gesellschaft verortet (Szene, Milieu, Revier). Eine „Tendenz“ o. ä., die den Linksextremismus mit der Mitte der Gesellschaft verbinden könnte, findet sich hier nicht - der Linksextremismus spielt sich sprachlich an den Rändern der Gesellschaft ab. Dem Linksextremismus werden sogar von den Akteuren reservierte Regionen im Stadtgebiet zugesprochen:

(5) [...] dass die Ausschreitungen auch deshalb besonders heftig werden, weil die Autonomen ihr Revier markieren wollen. (preG20)

Aus welchem Umfeld der Linksextremismus seine Akteure rekrutiert, wird in den Kollokaten nicht deutlich. 


\section{preNSU: Collocates}

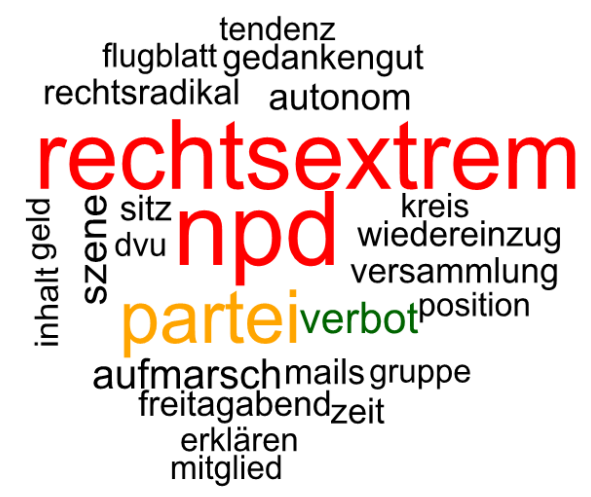

Abbildung 6: Kollokate für das LE-Feld RE vor der Veröffentlichung des NSU-Skandals (Mindestfrequenz: 3 )

\section{postNSU: Collocates}

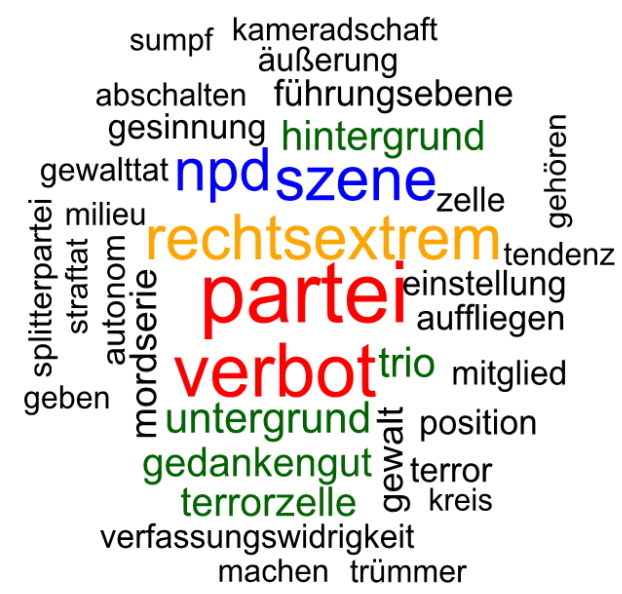

Abbildung 7: Kollokate für das LE-Feld RE nach der Veröffentlichung des NSU-Skandals (Mindestfrequenz: 5)

\subsection{Dimension: Ideologie und Organisation}

Die Rolle der Religion rückt bei post911 (islamisch) gegenüber pre911 (Gotteskrieger, Religionsunterricht) in den Hintergrund. Stattdessen wird der hohe Organisationsgrad der Akteure betont (Netzwerk, Organisation, Struktur). Ein Teil der Bedrohlichkeit speist sich aus der Unsichtbarkeit der Strukturen: 
(6) Er hatte am Montag nicht ausgeschlossen, dass sich weitere Mitglieder aus dem Netzwerk der al-Qaida in Deutschland aufhielten. (post911)

Ob die organisatorischen Strukturen nun für einen engeren oder einen loseren ideologischen Verbund stehen oder der Organisationsgrad nach den Ereignissen des 11. September schlicht neu bewertet wurde, könnte erst nach einer detaillierteren Analyse des Textmaterials beantwortet werden.

Weder aus den Kollokaten für preNSU noch für postNSU erfahren wir Genaueres über die ideologischen Merkmale des Rechtsextremismus. Da es sich um ein quantitativ gesehen vieldiskutiertes Phänomen handelt, ist davon auszugehen, dass in der medialen Kommunikation Grundkenntnisse zum Rechtsextremismus bei den Rezipienten vorausgesetzt werden. Die Kollokate deuten allerdings darauf hin, dass es sich um eine Weltanschauung bzw. Gesinnung handelt (Gesinnung, Einstellung), die graduell vorkommen kann (Tendenz; vgl. Textbeispiele (3) und (4)). Genauer zu untersuchen wäre, inwiefern das Framing von Rechtsextremismus als „Tendenz“ im jeweiligen Kontext mahnend oder verharmlosend wirkt.

Linksextremismus ist nach der Darstellung in postG20 keine einheitliche Ideologie, sondern umfasst verschiedene Varianten (Spektrum). Kulturelle Aspekte erscheinen für den Linksextremismus identitätsstiftend, bis hin zur Verknüpfung mit einer Musikform (Kulturzentrum, Punkband). Entgegen der Darstellung im Verfassungsschutzbericht, der betont, dass ein „herrschaftsfreies“ System Teil der linksextremen Ideologien ist, bilden die Subkorpora preG20 und postG20 einen gewissen Organisationsgrad des Linksextremismus ab (Zentrum, Struktur). Die folgende Formulierung etwa spiegelt Aussagen der sachsen-anhaltischen AfD-Landtagsfraktion wider:

(7) Man wolle untersuchen, welche linksextremistischen Strukturen in Sachsen-Anhalt bestehen und welche Aktivitäten diese entfalten. (preG20) 
preG20: Collocates

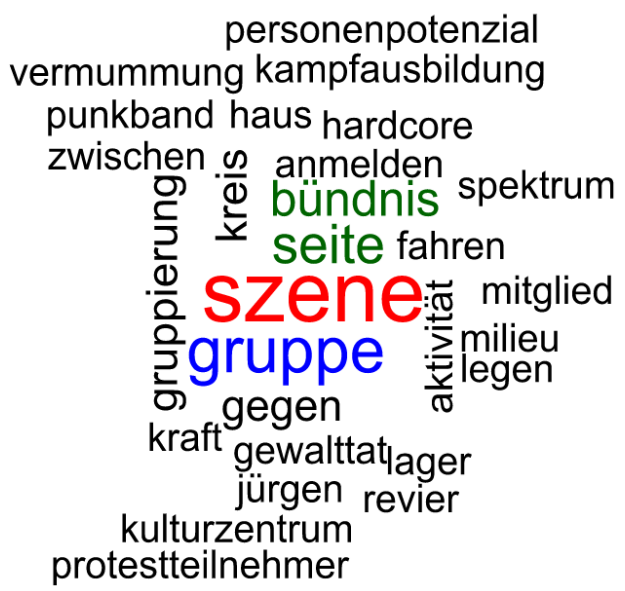

Abbildung 8: Kollokate für das LE-Feld LI vor dem G20-Gipfel (Mindestfrequenz: 1)

postG20: Collocates

Abbildung 9: Kollokate für das LE-Feld LI nach dem G20-Gipfel (Mindestfrequenz: 2)

\subsection{Dimension: Stellung zur Gesellschaft}

Die Kollokate von pre911 und post911 geben keine Hinweise darauf, wie der Islamismus zur Mehrheitsgesellschaft steht. Dass er einen grundlegenden Umbau der Gesellschaft zum Ziel hat, deutet sich jedoch an (radikal,fanatisch). Darauf weist auch die Thematisierung eines anstehenden 'Kampfes' hin:

(8) Als Nawar allerdings am Telefon seinen Kampfnamen "Saif" (das Schwert) nennt, [...]. Nach Recherchen der "SZ" ist er als 
radikaler Islamist schon länger unter Beobachtung und soll in der Vernehmung am Dienstag zugegeben haben, dass er mehrmals in Pakistan und auch in einem Trainingslager in Afghanistan war. (post911)

Beim Rechtsextremismus weisen einige Kollokate (Gesinnung, Tendenz) darauf hin, dass der Rechtsextremismus sowohl ein Phänomen der „Mitte der Gesellschaft“ ist als auch eines, das sich absondert (Szene, Milieu). Dies wirft die Frage auf, inwiefern einige der vom Rechtsextremismus propagierten Positionen kompatibel mit den Werten der bürgerlichen Gesellschaft sind, etwa die Betonung der öffentlichen Ordnung. So findet sich in einem Beitrag zu einem Gerichtsverfahren über Mitglieder der Gruppe "Sturm 34" folgender Abschnitt:

(9) Der Vorsitzende Richter Peter Lames sagte in der Urteilsbegründung, Ziel sei es gewesen, Mittweida "zeckenfrei und braun" zu machen. Die Organisation sei gegen alle vorgegangen, "die keine ausgesprochen rechtsradikale Orientierung" gehabt hätten. (preNSU)

Der hier zitierte Ausdruck zeckenfrei deutet ein vorgeblich „parasitäres Verhalten“ an. Dagegen vorzugehen, könnte über rechtsextreme Kreise hinaus auf Zustimmung treffen.

Bezüglich der Stellung des Linksextremismus zur Gesellschaft lässt sich aus den Kollokaten für preG20 und postG20 nur wenig ableiten. Dass Linksextremismus aus sprachlicher Sicht an den Rändern der Gesellschaft stattfindet, wurde bereits herausgearbeitet; hieraus leitet sich auch eine Inkompatibilität mit zentralen Aspekten der Mehrheitsgesellschaft ab. Anarchische Züge lassen sich in den lexikalischen Einheiten des LE-Felds LI identifizieren (autonom), nicht aber in den Kollokaten.

\subsection{Dimension: Typische Handlungen}

In beiden 911-Subkorpora ist die Assoziation des Islamismus mit Gewaltbereitschaft bis hin zu Terror abzulesen (pre911: Terrorismus, Gotteskrieger, radikal; post911: Terrorist, Terrorgruppe, Anschlag). Das entsprechende Framing war also bereits vor dem 11. September angelegt. Die neue Qualität besteht insbesondere darin, dass entsprechende Handlungen nicht unkoordiniert von einer „Gruppe“, sondern von Einheiten mit hohem Organisationsgrad begangen werden, die zum Äußersten bereit sind (radikal, fanatisch; vgl. Textbeispiel (8)).

Die Analyse der Kollokate des LE-Felds RE ist aufschlussreicher im Hinblick auf die typischen Handlungen in preNSU und postNSU. Besonders bezeichnend ist, dass Gewalt im Allgemeinen (Gerealt, 
Gerealttat) und gewalttätige Handlungen im Speziellen (Mordserie) erst nach dem NSU-Skandal systematisch mit Rechtsextremismus assoziiert werden. Auch dass Gewalt systematisch eingesetzt wird, um Schrecken zu verbreiten (Terror), scheint erst in postNSU deutlich ins Blickfeld zu rücken. Vor dem NSU-Skandal spielt lediglich der Aufmarsch als Aktivität eine prominente Rolle. Die Verknüpfung von Gewalt mit Rechtsextremismus hat in preNSU entweder einen regionalen Fokus auf Ostdeutschland, siehe (10) und (11), oder ist auf spezifische Antagonisten bezogen, siehe (12).

(10) Besonders auffällig ist erneut die Konzentration rechtsextremer Gewalt im Osten Deutschlands. (preNSU)

(11) Entgegen dem Bundestrend ist dort die Zahl der Taten angestiegen, von den insgesamt 762 rechtsextremen Gewalttaten entfielen allein 304 auf die fünf Ost-Länder. (preNSU)

(12) Der Innenminister warnte bei Präsentation des Verfassungsschutzberichts vor einer Spirale der Gewalt zwischen rechtsextremen Neonazis und linksextremen Autonomen. (preNSU)

Im Vergleich dazu entwickelt die Konzeption des Rechtsextremismus nach der Aufdeckung des Nationalsozialistischen Untergrunds eine neue Qualität durch die klare Assoziation mit gewalttätigem Handeln.

Aus den Kollokaten der beiden G20-Subkorpora spricht eine hohe Gewaltorientierung des Linksextremismus (Gewalttat). Gewalt ist nach dieser Darstellung kein Nebenprodukt, sondern wird gezielt eingesetzt (Vermummung). Für den Einsatz von Gewalt wird demnach sogar ausgebildet (Kampfausbildung). Diesbezüglich gibt es bereits eine Erwartungshaltung seitens der Beobachtenden (Potenzial), wobei der G20-Gipfel schon im Vorfeld als mögliches gewaltauslösendes Ereignis identifiziert wird:

(13) Allerdings habe es den Personen im Jahr 2016 an Ereignissen gefehlt, was weniger linksextremistische Straf- und Gewalttaten zur Folge hatte. Mit Blick auf den bevorstehenden G20-Gipfel und die Bundestagswahl im September dürfte sich dies ändern, heißt es in dem Bericht. (preG20)

Die Assoziation von Linksextremismus und Gewaltorientierung scheint also bereits vor dem G20-Gipfel eindeutig vorhanden zu sein. 


\subsection{Vorher-Nachher-Bild}

Bei zwei der drei Ereignisse können Veränderungen in der medialen Darstellung beobachtet werden, allerdings mit unterschiedlichen Schwerpunkten und unterschiedlichem Schweregrad.

Die Darstellung des Islamismus unterscheidet sich darin, dass nach dem 11. September der hohe Organisationsgrad der Akteure hervorgehoben wird. Eine substanzielle Änderung einer der vier als zentral angenommenen Dimensionen konnte allerdings nicht festgestellt werden. Ganz eindeutig war eine massive Frequenzveränderung der Instanzen des LE-Felds IM zu beobachten: Nach 9/11 treten Instanzen aus IM um fast das Zwanzigfache häufiger auf.

Die Darstellung des Rechtsextremismus ändert sich hingegen deutlich in postNSU im Gegensatz zu preNSU. Mit Blick auf Herkunft der Akteure und Stellung zur Gesellschaft rückt nach der Aufdeckung des Nationalsozialistischen Untergrundes in den Vordergrund, dass rechtsextreme Parallelstrukturen in eigenen Milieus oder gar im Untergrund existieren. Zudem wird der Rechtsextremismus mit einer Reihe neuer Typischer Handlungen von allgemeiner Gewalt bis hin zu spezifischer Gewalt wie Terror oder Mord assoziiert. Auch die Frequenz der Instanzen aus RE erhöht sich, der Faktor ist aber weitaus geringer im Vergleich zum Islamismus. Rechtsextremismus war also bereits vor dem NSU-Skandals ein als bedeutsam wahrgenommenes Thema.

Mit Blick auf den Linksextremismus lassen sich für die Zeit vor und nach dem G20-Gipfel keine nennenswerten Unterschiede feststellen. Weder ändert sich die Perspektive auf den Linksextremismus inhaltlich, noch lässt sich ein nennenswerter Anstieg der Berichterstattung verzeichnen. Dies ist zweifellos durch die Art des Ereignisses bedingt, das neben der insgesamt geringeren Bedeutung schon im Vorfeld bekannt war und bei dem gewaltsame Ausschreitungen zudem erwartet wurden.

\subsection{Vergleich der Extremismuskonzeptionen beim Verfassungs- schutz und bei SPIEGEL Online}

An dieser Stelle vergleichen wir die einleitend dargestellten Kerndefinitionen des Verfassungsschutzes (Abschnitt 2.2) mit den aus der Analyse hergeleiteten Konzeptionen der Extremismen in der medialen Darstellung von SPON.

Für den Bereich des Islamismus sind keine gravierenden Unterschiede zu erkennen. In beiden Fällen wird die religiös motivierte Ideologie ebenso thematisiert wie die Gewaltbereitschaft und die Zielsetzung, die Gesellschaft grundlegend umzubauen. Einzig bezüg- 
lich der Herkunft der Akteure deutet sich ein Unterschied an: Werden Akteure in SPON inklusive der Routen in unsere Gesellschaft benannt, äußert sich der Verfassungsschutzbericht hierzu nicht.

Der Linksextremismus hingegen wird sichtlich unterschiedlich dargestellt. In der Darstellung von SPON werden Netzwerkstrukturen und kulturelle Bezugspunkte benannt, während dies vom Verfassungsschutz in der Kerndefinition ausgespart wird. Die ideologischen Bezugspunkte werden vom Verfassungsschutz hingegen klar hervorgehoben, ebenso wie das Ziel, eine „herrschaftsfreie“ Gesellschaftsform zu etablieren; die Darstellung in SPON bleibt hier diffus, möglicherweise wird die Kenntnis dieser Zusammenhänge bei den Lesern vorausgesetzt. Die gesellschaftliche Randstellung der linksextremistischen Akteure wird in SPON durch Verortung in Szenen bzw. Milieus betont, der Verfassungsschutz thematisiert dies in seiner Charakterisierung des Linksextremismus nicht.

Ähnlich wie im Falle des Linksextremismus scheint SPON die Kenntnis ideologischer Eckpfeiler des Rechtsextremismus vorauszusetzen; der Verfassungsschutz geht detailliert auf Kernpunkte rechtsextremer Ideologie ein. Bezüglich der Verortung der Akteure lässt sich bei SPON klar die Idee des Spektrums von der Mitte bis zu den Rändern (bzw. „Untergrund“) erkennen, der Verfassungsschutz positioniert sich hierzu nicht klar. Uneinigkeit scheint mit Blick auf die Frage zu bestehen, inwiefern Gewaltbereitschaft ein zentrales Charakteristikum des Rechtsextremismus ist: In der Folge des NSUSkandals ist Gewalt ein wichtiges Element des Framings bei SPON, der Verfassungsschutz dagegen übernimmt diesen Aspekt auch Jahre nach der Aufdeckung des Nationalsozialistischen Untergrunds nicht in seine Kerndefinition.

\section{Diskussion und Ausblick}

In der vorliegenden Studie wurde am Beispiel von SPIEGEL Online untersucht, inwiefern sich das Framing von Extremismus nach einschneidenden Ereignissen ändern kann. Eine substanzielle Änderung des Framings war nur in einem Fall zu erkennen, nämlich für den Rechtsextremismus. In den beiden anderen Fällen war zumindest die Qualität des Framings im Vorfeld im Kern festgelegt, wobei im Falle des Islamismus ein massiver Anstieg der Berichterstattung nach den Anschlägen vom 11. September 2001 zu verzeichnen war.

Für den Rechtsextremismus ist zu vermuten, dass in der Phase vor der Veröffentlichung des NSU-Skandals die Zielsetzung und Gewaltbereitschaft der Akteure nicht erkannt wurde. Die daraus resultierende Darstellung in einem Leitmedium wie SPON war nicht dazu geeignet, Rechtsextremismus als sicherheitsrelevantes Phänomen 
mit konkretem Problemdruck in der öffentlichen Wahrnehmung zu verankern. Während hier in der Folge des NSU-Skandals schnell eine Veränderung im Framing zu verzeichnen ist, ist es umso erstaunlicher, dass der Verfassungsschutzbericht von 2017 Gewalt und Terror in der als „Überblick“ betitelten Kerndefinition (Bundesministerium des Innern, für Bau und Heimat 2017, S. 44) nicht als Merkmal des Rechtsextremismus in den Vordergrund rückt, wiewohl im Verlauf des Berichts das Gewaltpotenzial rechtsextremistischer Akteure inklusive des Phänomens Rechtsterrorismus immer wieder diskutiert wird. Dies steht in klarem Gegensatz zur Charakterisierung des Linksextremismus und des Islamismus durch den Verfassungsschutz. Daraus leitet sich die Frage ab, inwiefern der Rechtsextremismus durch den Verfassungsschutz auch Jahre später eher als weltanschaulich agitierender Extremismus verstanden wurde.

Darauf aufbauend stellt sich die Frage, inwiefern Unterschiede in der Konzeption Einfluss auf politisches Handeln haben. Wird bspw. der Islamismus plurizentrisch mit lokalen Einfallstoren bzw. als nur diffus zu verorten angesehen, so erscheint die Bedrohung ubiquitär und „dauerhafte Wachsamkeit" geboten. Wird hingegen der Rechtsextremismus als bis in die Mitte der Gesellschaft hinein hoffähig angesehen, könnte man daraus ableiten, dass eine entsprechende Aufklärungsarbeit nicht nur an „den Rändern der Gesellschaft“ ansetzen muss. Daher erscheint die Frage der Gewaltbereitschaft rechtsextremer Akteure geradezu zentral: Das tatsächliche Gefahrenpotenzial des Rechtsextremismus dürfte umso deutlicher hervortreten, je mehr die Konzeption auch auf der institutionellen Ebene Gewaltbereitschaft als zentrales Merkmal des Rechtsextremismus auffasst.

Auf methodischer Seite haben wir gezeigt, wie man Framingdurch-Assoziation für Korpora quantitativ auf Grundlage des Satzkontexts operationalisieren kann. Dabei ist die These, dass durch assoziatives Framing die patterns of invocation, also die Abrufmuster von Hintergrundinformation durch Rezipienten, potenziell beeinflusst werden können. Für diese These stützen wir uns auf Prinzipien der Fillmore'schen Framesemantik. Ebenfalls von der Framesemantik inspiriert ist die Analyse von LE-Feldern anstelle von einzelnen Suchwörtern: Da ein Erfahrungsbereich wie etwa eine bestimmte Extremismusvariante durch verschiedene sprachliche Elemente instanziiert werden kann, bietet es sich an, nicht nur Einzelelemente in die Framinganalyse einzubeziehen.

Da sich die vorliegende Studie auf den Zeitraum um ein besonders prominentes Ereignis beschränkt, sind lediglich Momentaufnahmen möglich. Wünschenswert wäre es, Framing- und Frequenzveränderungen über längere Zeiträume hinweg zu beobachten und damit mögliche Wendepunkte oder graduelle Verschiebungen in der 
Darstellung der Extremismen zu identifizieren. Außerdem sollte eine solche Analyse mehr als ein Medium umfassen, um mögliche weltanschauliche Einflüsse in der Berichterstattung berücksichtigen zu können.

Eine Langzeitstudie über die Begriffsentwicklung ließe außerdem $\mathrm{zu}$, Konjunkturen von Begrifflichkeiten zu erkennen, die weitere Hinweise auf das Framing von Ereignissen geben. Genannt sei hier das Beispiel des Begriffs Dönermorde, welcher die Mordserie des NSU in einen reinen Migrationskontext schob und der danach von SPON selbst thematisiert wurde. ${ }^{5}$ Bei einer Langzeitstudie könnte ein Vergleich von Framingmustern zu verschiedenen Zeitpunkten (z. B. mittels word embeddings, vgl. dazu Yao et al. 2018) helfen, größere Framingverschiebungen datenbasiert zu identifizieren.

Auch sollte die Analysegröße des LE-Felds weiterentwickelt werden. Einerseits ist noch ungeklärt, wie mit Dopplungen von lexikalischen Einheiten in den LE-Feldern und in den Kollokaten umzugehen ist (z. B. rechtsextremistisch sowohl im LE-Feld RE als auch in dessen Kollokaten), bzw. inwiefern solche Dopplungen auf die Analyse einen Einfluss haben. Neben der inhaltlichen Ebene wäre auch auf der Visualisierungsebene eine Weiterentwicklung wünschenswert, um die vielfältigen Beziehungen zwischen den Einheiten des LE-Felds und den Kollokaten aufzuzeigen.

Neben dieser methodischen Lücke ist auch das Textmaterial ausbaufähig, nicht nur mit Blick auf den medialen Diskurs. Wie bereits eingangs dargestellt, können Diskurse in sich strukturiert sein. Mit spezifischem Blick auf Extremismusdiskurse wäre die Frage, ob öffentlich-mediale und institutionelle Diskurse parallel verlaufen oder ob sie sich voneinander entkoppeln können und ob sich derartige Entwicklungen im Handeln der Institutionen widerspiegeln. Dies zu untersuchen ist unser Fernziel.

\section{Literatur}

Ackermann, Jan/Behne, Katharina/Buchta, Felix/Drobot, Marc/ Knopp, Philipp (2015): Metamorphosen des Extremismusbegriffes: Diskursanalytische Untersuchungen zur Dynamik einer funktionalen Unzulänglichkeit. Wiesbaden: Springer VS.

Baisa, Vít (2010): Towards Disambiguation of Word Sketches. In Sojka, Petr/Horák, Aleš/Kopeček Ivan/Pala, Karel (Hg.): Text, Speech and Dialogue. Berlin, Heidelberg: Springer Berlin Heidelberg, 37-42.

5 https://www.spiegel.de/panorama/gesellschaft/doener-mord-wie-das-unwortdes-jahres-entstand-a-841734.html (abgerufen am 23. September 2019). 
Benford, Robert D./Snow, David A. (2000): Framing processes and social movements: An overview and assessment. In: Annual reviere of sociology 26: 611-639.

Bönisch, Julia (2006): Meinungsführer oder Populärmedium? Das journalistische Profil von Spiegel Online. Berlin: Lit.

Bötticher, Astrid (2017): Radikalismus und Extremismus: Konzeptualisierung und Differenzierung zweier umstrittener Begriffe in der deutschen Diskussion. Leiden: Universität Leiden.

Bötticher, Astrid/Mareš, Miroslav (2012): Extremismus: Theorien Konzepte - Formen. München: Oldenbourg.

Bundesministerium des Innern, für Bau und Heimat (2017): Verfassungsschutzbericht 2017. Berlin: Bundesministerium des Innern, für Bau und Heimat.

Didakowski, Jörg/Geyken, Alexander (2014): From DWDS corpora to a German word profile: Methodological problems and solutions. In: Abel, Andrea/Lemnitzer, Lothar (Hg.): Vernetzungsstrategien, Zugriffsstrukturen und automatisch ermittelte Angaben in Internetwörterbüchern. Mannheim: Institut für Deutsche Sprache, 39-47.

Druckman, James N. (2001a): On the limits of framing effects: Who can frame? Journal of Politics 63: 1041-1066.

Druckman, James N. (2001b): The implications of framing effects for citizen competence. Political Behavior 23: 225-256.

Ebling, Sahra/Scharloth, Joachim/Dussa, Tobias/Bubenhofer, Noah (2014): Gibt es eine Sprache des politischen Extremismus? In: Liedtke, Frank (Hg.): Die da oben: Texte, Medien, Partizipation. Bremen: Hempen, 43-68.

Entman, Robert M. (1993): Framing: Toward clarification of a fractured paradigm. In: Journal of communication 43, 51-58.

Fillmore, Charles (1985) Frames and the semantics of understanding. In: Quaderni di Semantica 6, 222-254.

Fillmore, Charles J. (1982): Frame semantics. In: Linguistics in the morning calm. Seoul: Hanshin, 111-137.

Ghersetti, Marina (2014): Still the same? Comparing news content in online and print media. In:Journalism Practice 8, 373-389.

Jäger, Ludwig (2018). „Outthereness“. Über das Problem des Wirklichkeitsbezugs von Zeichen. In: Felder, Ekkehard/Gardt, Andreas (Hg.): Wirklichkeit oder Konstruktion? Sprachtheoretische und interdisziplinäre Aspekte einer brisanten Alternative. De Gruyter Mouton, 301-323.

Kepplinger, Hans Mathias/Geiss, Stefan/Siebert, Sandra (2012): Framing scandals: Cognitive and emotional media effects. In:Journal of Communication 62, 659-681.

Kilgarriff, Adam et al. (2014): The Sketch Engine: Ten years on. In: Lexicography 1, 7-36. 
Klein, Josef (2018): ,Betrachten der Wirklichkeit" und politisches Framing: Am Beispiel der CDU-Wahlkampagne 2013. In: Felder, Ekkehard/Gardt, Andreas (Hg.): Wirklichkeit oder Konstruktion? Sprachtheoretische und interdisziplinäre Aspekte einer brisanten Alternative. De Gruyter Mouton, 344-370.

Klein, Wolfgang/Geyken, Alexander (2010): Das „Digitale Wörterbuch der Deutschen Sprache DWDS“. In: Heid, Ulrich/Schierholz, Stefan/Schweickard, Wolfgang/Wiegand, Herbert Ernst/ Gouws, Rufus H./Wolski, Werner S. (Hg.): Lexicographica 26, 7996.

Klemperer, Victor (2018): LTI: Notizbuch eines Philologen. Ditzingen: Reclam.

Lasch, Alexander (2014): Zur Vereinbarkeit von diskurslinguistisch motivierter Sprachgeschichtsschreibung und maschineller Sprachanalyse am Beispiel des „Islamismus“-Diskurses. In: Ágel, Vilmos/Garde, Andreas (Hg.): Paradigmen der aktuellen Sprachgeschichtsforschung. Berlin: de Gruyter, 231-249.

Liebert, Wolf-Andreas (2019): Zur Sprache totaler Ideologien: Wie die Linguistik zum Verstehen extremistischen Denkens und Sprechens beitragen kann. In: Sprachreport 35, 1-12.

Meyer-Lucht, Robin (2007): Das Wunder von Hamburg: Wie es ausgerechnet Spiegel Online gelang, sich als Deutschlands führende Nachrichtensite durchzusetzen. In: Friedrichsen, Mike/Brunner, Martin F. (Hg.): Perspektiven für die Publikumszeitschrift. Berlin: Springer, 83-97.

Mletzko, Matthias (2010): Gewalthandeln linker und rechter militanter Szenen. In: Aus Politik und Zeitgeschichte 44, 9-16.

Scharloth, Joachim/Eugster, David/Bubenhofer, Noah (2013): Das Wuchern der Rhizome: Linguistische Diskursanalyse und Datadriven Turn. In: Busse, Dietrich/Teubert, Wolfgang (Hg.): Linguistische Diskursanalyse: neue Perspektiven. Wiesbaden: VS, 345380.

Schuck, Andreas R. T./De Vreese, Claes H. (2006): Between risk and opportunity: News framing and its effects on public support for EU enlargement. In: European Journal of Communication 21, 532.

Schuppener, Georg (2010): Sprache des Rechtsextremismus: Spezifika der Sprache rechtsextremistischer Publikationen und rechter Musik. Leipzig: Edition Hamouda.

Vonbun-Feldbauer, Ramona/Matthes, Jörg (2018): Do channels matter? Investigating media characteristics in the agenda-building process of an election campaign. In:Journalism Studies 19, 23592378.

Yao, Zijun/Sun, Yifan/Ding, Weicong/Rao, Nikhil/Xiong, Hui (2018):

Dynamic Word Embeddings for Evolving Semantic Discovery. In: 
Proceedings of the Eleventh ACM International Conference on Web Search and Data Mining - WSDM 18, 673-681. DOI: 10.1145/3159652.3159703.

\section{Anhang}

Im Folgenden sind die Begriffe der drei LE-Felder gelistet. Scheinbare Mehrfachnennungen bilden entweder unterschiedliche Wortklassen ab (z. B. rechstextrem/Rechtsextrem) oder gleichen Lücken in der Lemmatisierung aus (z. B. Dschihadist|Dschihadisten). Die Kollokationstabellen zu den LE-Feldern sind für eine tabellarische Darstellung zu umfangreich und werden daher als separater Download bereitgestellt.

\section{LE-Feld IM}

\begin{tabular}{|l|l|}
\hline Al-Qaida & islamistisch \\
\hline Boko Haram & Jihadist \\
\hline Dschihad & Jihadisten \\
\hline Dschihadist & Muslimbruder \\
\hline Dschihadisten & Muslimbruderschaft \\
\hline dschihadistisch & Salafist \\
\hline Hamas & salafistisch \\
\hline Hassprediger & Taliban \\
\hline Hisbollah & Tugendpartei \\
\hline IS & Wahhabit \\
\hline Islamischer Staat & wahhabitisch \\
\hline Islamismus & Wohlfahrtspartei \\
\hline Islamist & \\
\hline
\end{tabular}




\section{LE-Feld LI}

\begin{tabular}{|l|l|l|}
\hline anti-imperialistisch & autonom & linksextrem \\
\hline antiimperialistisch & Autonome & linksextremistisch \\
\hline Antifa & DKP & linksradikal \\
\hline
\end{tabular}

\section{LE-Feld RE}

\begin{tabular}{|l|l|}
\hline Antisemit & nationalistisch \\
\hline antisemitisch & Nationalsozialist \\
\hline ausländerfeindlich & nationalsozialistisch \\
\hline braun & NPD \\
\hline Braun & NSU \\
\hline DVU & rechtsaußen \\
\hline Faschist & rechtsextrem \\
\hline faschistisch & Rechtsextrem \\
\hline faschistoid & Rechtsextreme \\
\hline fremdenfeindlich & rechtsradikal \\
\hline Hooligan & rechtsradikal \\
\hline Kameradschaft & Rechtsradikaler \\
\hline Nationaldemokrat & Skinhead \\
\hline Nationalist & Volksunion \\
\hline
\end{tabular}

